\title{
Research Paper: Problematic Smartphone Use and Aggressive Behavior Among University Students: The Mediating Role of Sleep Disturbance
}

\author{
Salman Zarei ${ }^{1^{*}}$ (D) \\ 1. Department of Psychology, Faculty of Literature and Humanities, Lorestan University, Khorramabad, Iran.
}

Citation Zarei S. Problematic Smartphone Use and Aggressive Behavior Among University Students: The Mediating Role of Sleep Disturbance. Journal of Research \& Health. 2021; 11(3):157-164. http://dx.doi.org/10.32598/JRH.11.3.1224.3

http://dx.doi.org/10.32598/JRH.11.3.1224.3

\section{Keywords:}

Sleep, Aggression, Smartphone

\section{ABSTRACT}

Background: Despite several benefits, research has found that problematic smartphone use is positively associated with behavioral problems. However, the internal mechanisms underlying this relationship need further investigation. So, the current study aimed to investigate the mediating role of sleep disturbance in the relationship between problematic smartphone use and aggression.

Methods: The research design was descriptive-correlational. Also, the study population included the male university students of the Islamic Azad University of South Tehran Branch in the academic year of 2019-2020. Using the convenience sampling method, a total number of 213 participants from two faculties (Faculty of Human Sciences and Faculty of Law and Political Sciences) were selected and tested by Buss-Perry Aggression Questionnaire, Smartphone Addiction Scale-Short Version, and Pittsburgh Sleep Quality Index. Then, the Pearson correlation and path analysis were conducted to analyze the data.

Results: The results revealed the significant and direct effects of problematic smartphone use on aggression $(\beta=0.12, \mathrm{P}<0.05)$, sleep quality on aggression $(\beta=0.37, \mathrm{P}<0.001)$, and problematic smartphone use on sleep disturbance $(\beta=0.42, \mathrm{P}<0.001)$. Also, sleep quality significantly mediated in the relationship between problematic smartphone use and aggression $(\beta=0.15, \mathrm{P}<0.01)$.

Conclusion: According to the present findings, problematic smartphone use and sleep disturbance are two important factors affecting aggressive behavior among university students. These findings highlight the critical role of early intervention for aggression with a focus on those with problematic smartphone use and more specifically those with sleep disturbance.

\section{"Corresponding Author:}

Salman Zarei, $P h D$.

Address: Department of Psychology, Faculty of Literature and Humanities, Lorestan University, Khorramabad, Iran

Phone: +98 (912) 8364266

E-mail:salman_zarei@yahoo.com 


\section{Introduction}

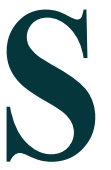

martphone use has significantly increased over the last decade, especially among university students [1]. Academic purposes, facilitating information access, and maintaining social relationships are smartphones' attractive attributes and characteristics [2] that could increase the potential for smartphone overuses [3]. Smartphone overuse behaviors are related to addiction-like symptoms, such as tolerance and loss of control, which have been described as problematic smartphone use [4]. Problematic smartphone use is defined as unsuitable or extreme smartphone use that might impede everyday life, lessen social functions, and lead to psychological and behavioral problems [5]. This problematic smartphone use has given rise to the term nomophobia that is defined as an uncontrollable fear of leaving home without a mobile phone and causes symptoms, such as anxiety, emotional instability, aggressiveness, and difficulty concentrating [6]. Studies carried out among university students showed that between $42 \%$ to $66 \%$ of smartphone users suffer from nomophobia [7]. Research in college students has also found that problematic smartphone use can cause several detrimental consequences, including sleep interference [8], aggression [9], poor academic performance [10], negative emotions [2], and mobile phone-based aggression [11]. Also, Visnjic et al. found that the overuse of smartphones could negatively affect the mental health of university students [12]. Accordingly, university students were found to be seriously impotent to the negative health impacts of problematic smartphone use [13]. In addition, empirical studies indicate that college students with problematic smartphone use often exhibit antisocial behaviors, such as poor peer relationships, suicidal ideation, cyberbullying, and involving in crimes $[14,15]$.

Among the negative health impacts of problematic smartphone use, aggression has been the focus of attention among scholars. According to Buss "aggression is a feeling of anger or antipathy resulting in hostile or violent behavior" [16]. With the rapid popularity of the smartphone, its extreme use influenced various aspects of college students' study and life [17]. In this line, Um et al. reported that although smartphone use may reduce distress by providing immediate rewards and opportunities to engage in different activities, problematic smartphone use is an important risk factor for aggression [18]. Similarly, Yen et al. found a high correlation between aggressive behaviors and problematic smartphone use [19]. Furthermore, the studies of Mehroof and Griffiths [20] and Polman et al. [21] showed that problematic smartphone use was significantly correlated with aggression.

Another important variable that can drive aggressive behavior is sleep disturbance [22-24]. In the Diagnostic and Statistical Manual of Mental Disorders, Fifth Edition (DSM-5) sleep disorders encompass 10 conditions manifested by disturbed sleep and causing distress as well as impairment in daytime functioning. Disturbed sleep, whether due to quality and timing or duration can have many adverse health consequences. The most obvious concerns include fatigue and cognitive focus, also, mood can be greatly affected [25]. Based on previous studies sleep disturbance is a negative outcome of problematic smartphone use among university students [8]. In line with this, sleep quality is exacerbated with an increasing level of extreme smartphone use [26]. Furthermore, university students have to confront "leaving home", increased autonomy, alters in friend groups, and new social circumstances and handle educational responsibilities [27]. About $90 \%$ of university students have roommates, and among them, $41 \%$ wake up at night owing to the interference of others [28]. These issues and special situations faced by university students are related to sleep problems [29]. Gaultney found that $27 \%$ of all university students are at risk of at least one sleep disorder [30]. Naturally, students with poor sleep quality suffer from mental health problems, such as chronic fatigue, aggression, and negative emotions [28, 31]. The result of Lemma et al. study showed that among a large population of university students, 55.8\% (1424) had poor sleep quality and $34.1 \%$ (864) had aggressive behavior [32]. Also, Vali-Smith et al. discovered that university students who reported poor sleep quality significantly had one type of aggressive behavior [33].

Altogether, as mentioned before problematic smartphone use may pose many deleterious outcomes for university students. College students are considered as the early adopters of smartphones [34]. Hence, it is highly pertinent to focus on university students when examining problematic smartphone use. In this regard, some scholars argue that individuals navigating this developmental stage are more likely to engage in aggressive behavior [35]. Although an enormous amount of research is in line with the notion that high sleep disturbances could predict university students' aggressive behavior, minimal studies have directly tested the mediating role of sleep disturbance in the association between problematic smartphone use and aggressive behavior. The researcher expects this study to provide an in-depth understanding of problematic smartphone use and aggression among college students and contribute to the 
development of effective mental health interventions. To investigate the relationship between problematic smartphone use and aggression, problematic smartphone use was taken as an independent variable and aggression as a dependent variable to establish a path analysis model. So that, it was possible to investigate the mediating role of sleep disturbance in the relationship between problematic smartphone use and aggression to figure out the inner deep structure of the three variables. Above all, the current study aimed to examine problematic smartphone use and its association with aggressive behavior in university students. Furthermore, how sleep disturbance mediates this association is investigated.

\section{Methods}

This cross-sectional and correlational study was conducted on the students of Islamic Azad University, Tehran South Branch, Iran, in 2019. A total of 220 male university students from two faculties (Faculty of Human Sciences and Faculty of Law and Political Sciences) were selected by convenience sampling strategy. There is no consensus on the sample size for path analysis. However, many researchers recommended 200 cases as the minimum sample. The inclusion criteria were as follows: 1) the age range of 18 to 25 years; and 2) having more than two years of smartphone use experience (based on a preliminary interview and participants' selfreport). The only exclusion criterion was having a background of psychological problems (based on a preliminary interview and participants' self-report).

About the demographic characteristics of the participants, age Mean 20.62 years $(\mathrm{SD}=3.28)$. Moreover, 79 students $(37.1 \%)$ were freshmen, 52 students $(24.4 \%)$ were sophomores, 61 students $(28.6 \%)$ were junior, and 21 students $(9.90 \%)$ were senior. Before completing the formal survey, all participants gave their written informed consent. Also, they were informed that the investigation was anonymous and confidential and participants had the right to withdraw at any time without explanation. Next, the researcher distributed the paperand-pencil questionnaires and collected them, in the regular classrooms. The following instruments were used to collect the data.

Buss-Perry Aggression Questionnaire (BPAQ): The BPAQ is a 29-item instrument that measures aggressive behaviors. The questionnaire uses a Likert-type response format ranging from 1 (extremely uncharacteristic of me) to 5 (extremely characteristic of me), yielding a minimum score of 29 and a maximum score of 145 [16]. The internal consistency coefficients for the subscales were as follows: physical aggression, $\alpha=0.85$; verbal aggression, $\alpha=0.72$; anger, $\alpha=0.83$; and hostility, $\alpha=0.77$; with the internal consistency being $\alpha=0.89$. Besides, the test-retest reliability (nine weeks) for the subscales and total score ranged from $\alpha=0.72$ to $\alpha=0.80$ [36]. In the present study, the Persian translation of the BPAQ demonstrated good internal consistency with the Cronbach $\alpha$ value of 0.85 .

Smartphone Addiction Scale-Short Version (SAS-SV): The SAS-SV measures problematic smartphone use severity. The SAS-SV contains 10 items with response options from $1=$ strongly disagree to $6=$ strongly agree. The overall score for the scale ranges from 10 to 60 , wherein higher scores show higher levels of problematic smartphone use [37]. Studies demonstrate scale reliability, and scores converge with other measures of PSU and PIU and smartphone use frequency [38]. In the present study, the Persian translation of the SAS-SV demonstrated good internal consistency with the Cronbach $\alpha$ value of 0.91 .

Pittsburgh Sleep Quality Index (PSQI): The PSQI is a self-rated scale that assesses sleep quality during the past month. The scale contains 18 items, which covered seven sleep quality components. Each component scored from 0 (no difficulty) to 3 (severe difficulty). A total score was totaled from the seven component scores, ranging from 0 to 21, with the score of 5 used as the cutoff point. A total score less than or equal to 5 was recognized as good sleep, while a score of more than 5 implied poor sleep quality [39]. Mollayeva et al. performed a meta-analysis and found strong evidence for the PSQI's reliability and validity [40]. In the present study, the Persian translation of the PSQI demonstrated good internal consistency with the Cronbach $\alpha$ value of 0.88 .

Means, standard deviations, and Pearson correlations for the levels of aggression, sleep quality, and problematic smartphone use together were conducted using SPSS v. 23. Also, path analysis was conducted to examine the mediating role of sleep quality using AMOS v. 21.0. It is noted that seven participants missed more than $50 \%$ of items on a given scale; they were excluded from subsequent analyses.

\section{Results}

Table 1 presents descriptive statistics, such as mean, standard deviation, and correlation coefficients. The result of the Kolmogorov-Smirnov test confirmed the normal distributions of the study variables $(\mathrm{P}>0.05)$. Also, the correlation coefficients (Table 1) showed that all relationships were significantly positive at $\mathrm{P}<0.01$. Therefore, the significant relationship between the vari- 
Table 1. Descriptive statistics, correlation matrix, and normality tests for the study variables

\begin{tabular}{cccccccc}
\hline Variables & Mean \pm SD & $\mathbf{1}$ & $\mathbf{2}$ & $\mathbf{3}$ & \multicolumn{2}{c}{ Kolmogorov-Smirnov } \\
\cline { 5 - 7 } & & & & & Statistics & $\mathbf{P}$ \\
\hline 1. Problematic smartphone use & $36.07 \pm 6.39$ & 1 & - & - & 0.073 & 0.061 \\
2. Sleep disturbance & $8.93 \pm 3.07$ & $0.51^{* *}$ & 1 & - & 0.059 & 0.082 \\
3. Aggression & $76.52 \pm 10.61$ & $0.24^{* *}$ & $0.41^{* *}$ & 1 & 0.079 & 0.055 \\
\hline
\end{tabular}

${ }^{* *} \mathrm{P}<0.01$.

DRA

ables satisfied the necessary condition for investigating the mediating role of sleep quality in the relationship between problematic smartphone use and aggression.

Before conducting path analysis, it was ensured that its basic assumptions, such as adequacy of the sample size, data distribution normality, and multicollinearity were established. Table 1 reports the research model of the relationship between problematic smartphone use and aggressive behavior, which is mediated by sleep quality, based on standardized coefficients $(\beta)$. According to Table 2, the proposed model has a good fit based on the fitness indices. The most commonly used fitness index in the model analysis is Chi-square/ Degree of Freedom (CMIN/DF), which shows a good fit with a value of less than 3 in the current research model. The Goodness of Fit Index (GFI), the Normalized Fit Index (NFI), and the Comparative Fit Index (CFI) with values of higher than 0.90 indicate an acceptable fit to the current data. Also, the Root Mean Square Error of Approximation (RMSEA) with a value of between 0.05 and 0.10 shows an acceptable fit of the model (Figure 1).

Table 3 presents the parameters of the direct and indirect relationship measurement in the research model. As shown, problematic smartphone use significantly and directly affected aggression $(\beta=0.12$, Critical Ratio (C.R) $=2.64, \mathrm{P}<0.01)$ and sleep quality $(\beta=0.42, \mathrm{C} . \mathrm{R}=7.89$,
$\mathrm{P}<0.001)$. Moreover, the effect of sleep quality were significant on aggression $(\beta=0.37, C . R=4.51, P<0.001)$.

Finally, the result of the bootstrap method for investigating indirect pathway (Table 4) shows that the upper (0.19) and lower (0.09) limits of the indirect relationship of the problematic smartphone with aggressive behavior did not include zero, indicating a significant indirect pathway $(\beta=0.15, \mathrm{t}=2.30, \mathrm{P}<0.01)$.

\section{Discussion}

The present study investigated the mediating role of sleep quality in the relationship between problematic smartphone use and aggressive behavior among university students. The results showed that problematic smartphone use significantly and positively affects aggressive behavior in university students; this is consistent with the results of previous research $[17,21]$. Accordingly, Um et al. [18] and Yen et al. [19] reported that problematic smartphone use is an important risk factor for aggression, and the frequency of cyber aggression is significantly high among students who excessively use a smartphone. The current finding is also in line with Mehroof and Griffiths's [20] study showing that smartphone dependency can result in behavioral problems, such as the feeling of anger, tension, aggression, and hostility among students. Because of some stressors and special

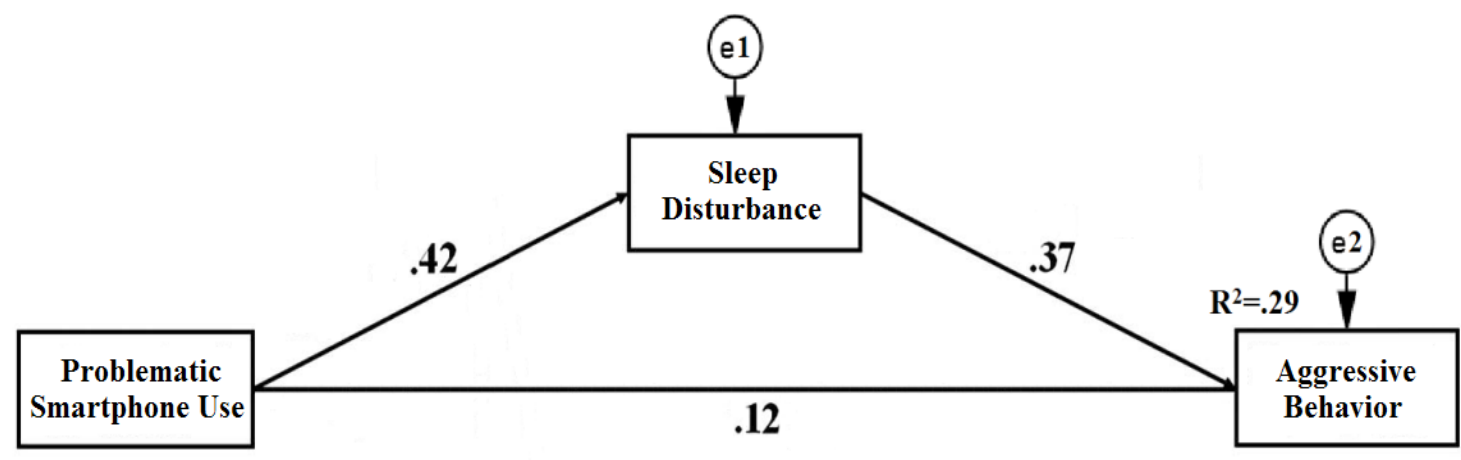

Figure 1. Model of the mediating role of sleep disturbance 
Table 2. General $\mathrm{ft}$ assessment indices of the model

\begin{tabular}{cccccc}
\hline Indices & CMIN/DF & GFI & SRMR & CFI & NFI \\
\hline Model values & 2.008 & 0.92 & 0.03 & 0.94 & 0.91 \\
Acceptable values & Less than 3 & Greater than 0.90 & Less than 0.05 & Greater than 0.90 & Greater than $0.90 \quad$ Less than 0.08 \\
Status & Good & Good & Good & Good & Good \\
\hline
\end{tabular}

MRt

CMIN/DF: Chi-square/Degree of Freedom; GFI: Goodness of Fit Index; NFI: Normalized Fit Index; CFI: Comparative Fit Index; RMSEA: Root Mean Square Error of Approximation; SRMR: Standardized Root Mean Square Residual.

circumstances in academic life, university students may suffer from mental health problems, such as chronic fatigue as a result of problematic smartphone use, and then lose their self-control in challenging situations [9]. In line with this, an explorative prospective study reported that aggressive behavior worsened with a high lack of self-control [13]. Another possible explanation is that according to the social learning theory, individuals can get aggressive behaviors unconsciously and reduce the inhibition of aggression in the internet violence through a smartphone for a long time so that the aggression will be strengthened [15].

The findings showed that problematic smartphone use significantly affected aggressive behavior through sleep quality; this is in line with other studies [22, 23]. One hypothesis is that many technologies, such as smartphones could produce shortwave light [8]. When many students use smartphones at night, artificial shortwave light exposure could influence sleep and neurobehavioral functions. Also, chronic inopportune exposure to shortwave light causes a malfunction of the circadian timing system, which results in sleep disturbances [26]. Accordingly, some studies revealed that college students use their smartphones while in bed before falling asleep [28]. Hence, one explanation is that university students want to prolong their time online, which results in a sleep phase shift to later hours. In addition, excessive smartphone use leads to sleep disturbances due to irregular bedtime patterns [30]. Studies showed that individuals with low sleep quality are vulnerable to the negative effects of poor sleep quality, such as aggressive behavior [28].

Poor sleep quality affects emotional functioning and is accompanied by emotional instability, greater irritability, and short-temperedness that may result in outbursts of aggression [32]. Moreover, individuals who experience serious sleep problems reported physical aggressive behaviors in their interpersonal relationships [31]. Also, according to Coulombe et al. sleep problems contribute to the loss of emotional control, including the regulation of aggression [24]. Accordingly, Polman et al. found that in confrontation with a frustrating situation, college students who reported the overuse of smartphones and insufficient sleep time, because of the lack of affect control and frustration tolerance, had significantly higher levels of aggression, compared with those with enough sleep time [21]. A plausible explanation for the mediating effects of sleep disturbance may be that problematic smartphone use can disrupt university students' normal sleep patterns; this contributes to a high risk of aggression [25]. Another explanation is that sleep deprivation results in poor prefrontal cortical functioning. When prefrontal cortical functioning is reduced, the ability to impulse control and initiate behavioral responses based on cognitive and social context diminished, which could potentially lead to the uncontrolled, impulsive, and outward expression of aggressive responses [33].

Several limitations should be noted when interpreting this study's findings. First, the present study only inves-

Table 3. Summary of direct relationship measurement parameters in the general research model

\begin{tabular}{ccccccc}
\hline Direct Pathways & B & SE & Beta & C.R & P \\
\hline Problematic smartphone use $\rightarrow$ Aggressive behavior & 0.609 & 0.011 & 0.121 & 0.104 & 0.05 \\
Problematic smartphone use $\rightarrow$ Disturbance & 0.017 & 0.002 & 0.423 & 7.891 & 0.001 \\
Sleep disturbance $\rightarrow$ Aggressive behavior & 0.281 & 0.054 & 0.371 & 4.513 \\
\hline
\end{tabular}


Table 4. Results of the bootstrap method of indirect pathway in the general research model

\begin{tabular}{cccccc}
\hline Model Pathway & Beta & SE & T & \multicolumn{2}{c}{ (95\%Cl) } \\
& & & & Lower Bound & Upper Bound \\
\hline $\begin{array}{c}\text { Problematic smartphone use } \rightarrow \text { Sleep disturbance } \\
\rightarrow \text { Aggressive behavior }\end{array}$ & 0.156 & 0.207 & 2.301 & 0.095 & 0.193 \\
\hline
\end{tabular}

tigated the students at the Islamic Azad University of Tehran, therefore, may not represent the total population. Second, the cross-sectional design, which is not the best way to evaluate causal relations, also limits the results. Future longitudinal research or experimental designs are needed to further ascertain the particular directions and relationships among these variables. Third, all of the measures were self-reports, which might have influenced the study's validity. Future studies could employ multiple methods (e.g. standardized interview and observation) to collect data, thereby, providing more detailed information and reducing potential common method bias. Finally, the literature in this field is not yet rich enough.

This study is innovative in exploring sleep disturbance as a mediating variable; this has not received previous scrutiny. Limitations aside, the present study provided some theoretical and practical implications. The findings of this study could help to guide targeted preventions and interventions for aggressive behavior in university students. Thus, mental health practitioners can educate students about the potential harms of poor sleep quality and problematic smartphone use and guide them to set self-reinforcing goals to monitor their frequency and duration of smartphone use. These strategies may be instrumental in helping them to overcome aggressive behaviors efficiently and regulate themselves properly, promoting healthy ways of using smartphones. Furthermore, this study expands our knowledge on the aggressive behaviors associated with high problematic smartphone use and highlights the role of poor sleep quality in setting undesirable conditions for university students.

\section{Conclusion}

This study tested a mediation model to examine whether sleep quality underlies the relationship between problematic smartphone use and aggressive behavior. The results revealed that the problematic use of smartphones was a risk factor for poorer sleep quality in a sample of university students, and predicted aggressive behavior through the sleep quality. These findings substantially contribute to our understanding of problematic smart- phone use and aggressive behavior. This likelihood demonstrated the superposition effects of the two risk factors. Results suggest that intervention could decrease the level of problematic smartphone use and poor sleep quality, which in turn decrease the level of aggression. Also, university students displaying high aggressive behavior should be attentively monitored for smartphone overuse. Ultimately, many students neglect to consider the negative aspects of smartphones, such as the waste of time, the occupation of cognitive and psychological capacities, dependence on cell phones, and the anxiety due to the variety of mobile applications. Therefore, to reduce the negative effects of the excessive use of smartphones on students' sleep, it is necessary to provide university students with information about the direct and indirect negative effects of the excessive use of smartphones on aggression.

\section{Ethical Considerations}

\section{Compliance with ethical guidelines}

This study was approved by the Ethics Committee of Islamic Azad University, Tehran Medical Branch (Ethic code: IR.IAU.TMU.REC.1398.161.2). All ethical principles are considered in this article. The participants were informed about the purpose of the research and its implementation stages. They were also assured about the confidentiality of their information and were free to leave the study whenever they wished, and if desired, the research results would be available to them.

\section{Funding}

This research did not receive any specific grant from funding agencies in the public, commercial, or not-forprofit sectors.

\section{Conflict of interest}

The author declared that he had no conflict of interest. 


\section{References}

[1] Elhai JD, Contractor AA. Examining latent classes of smartphone users: Relations with psychopathology and problematic smartphone use. Comput Hum Behav. 2018; 82:159-66. [DOI:10.1016/j.chb.2018.01.010]

[2] Elhai JD, Dvorak RD, Levine JC, Hall BJ. Problematic smartphone use: A conceptual overview and systematic review of relations with anxiety and depression psychopathology. J Affect Disord. 2017; 207:251-9. [DOI:10.1016/j.jad.2016.08.030] [PMID]

[3] Castellacci F, Tveito V. Internet use and well-being: A survey and a theoretical framework. Res Pol. 2018; 47(1):308-25. [DOI:10.1016/j.respol.2017.11.007]

[4] Chun J. Conceptualizing effective interventions for smartphone addiction among Korean female adolescents. Child Youth Serv Rev. 2018; 84:35-9. [DOI:10.1016/j.childyouth.2017.11.013]

[5] Billieux J, Maurage P, Lopez-Fernandez O, Kuss DJ, Griffiths MD. Can disordered mobile phone use be considered a behavioral addiction? An update on current evidence and a comprehensive model for future research. Curr Addict Rep. 2015; 2(2):156-62. [DOI:10.1007/s40429-015-0054-y]

[6] Arpaci I. Culture and nomophobia: The role of vertical versus horizontal collectivism in predicting nomophobia. Inf Dev. 2019; 35(1):96-106. [DOI:10.1177/0266666917730119]

[7] Yildirim C, Correia AP. Exploring the dimensions of nomophobia: Development and validation of a self- reported questionnaire. Comput Hum Behav. 2015; 49:130-7. [DOI:10.1016/j.chb.2015.02.059]

[8] Xie X, Dong Y, Wang J. Sleep quality as a mediator of problematic smartphone use and clinical health symptoms. J Behav Addict. 2018; 7(2):466-72. [DOI:10.1556/2006.7.2018.40] [PMID] [PMCID]

[9] Cho HY, Kim DJ, Park JW. Stress and adult smartphone addiction: Mediation by self-control, neuroticism, and extraversion. Stress Health. 2017; 33(5):624-30. [DOI:10.1002/ smi.2749] [PMID]

[10] Samaha M, Hawi NS. Relationships among smartphone addiction, stress, academic performance, and satisfaction with life. Comput Hum Behav. 2016; 57:321-5. [DOI:10.1016/j. chb.2015.12.045]

[11] Rozgonjuk D, Levine JC, Hall BJ, Elhai JD. The association between problematic smartphone use, depression and anxiety symptom severity, and objectively measured smartphone use over one week. Comput Hum Behav. 2018; 87:10-7. [DOI:10.1016/j.chb.2018.05.019]

[12] Višnjić A, Veličković V, Sokolović D, Stanković M, Mijatović $\mathrm{K}$, Stojanović M, et al. Relationship between the manner of mobile phone use and depression, anxiety, and stress in university students. Int J Environ Res Public Health. 2018; 15(4):697. [DOI:10.3390/ijerph15040697] [PMID] [PMCID]

[13] Vally Z, El Hichami F. An examination of problematic mobile phone use in the United Arab Emirates: Prevalence, correlates, and predictors in a college-aged sample of young adults. Addict Behav Rep. 2019; 9:100185. [DOI:10.1016/j. abrep.2019.100185] [PMID] [PMCID]
[14] Wang P, Zhao M, Wang X, Xie X, Wang Y, Lei L. Peer relationship and adolescent smartphone addiction: The mediating role of self-esteem and the moderating role of the need to belong. J Behav Addict. 2017; 6(4):708-17. [DOI:10.1556/2006.6.2017.079] [PMID] [PMCID]

[15] Marchant A, Hawton K, Stewart A, Montgomery P, Singaravelu $\mathrm{V}$, Lloyd $\mathrm{K}$, et al. A systematic review of the relationship between internet use, self-harm and suicidal behavior in young people: The good, the bad and the unknown. PLoS One. 2017; 12(8):e0181722. [DOI:10.1371/journal.pone.0181722] [PMID] [PMCID]

[16] Buss AH, Perry M. The aggression questionnaire. J Pers Soc Psychol. 1992; 63(3):452-9. [DOI: 10.1037//0022-3514.63.3.452] [PMID]

[17] Wang JL, Wang HZ, Gaskin J, Wang LH. The role of stress and motivation in problematic smartphone use among college students. Comput Hum Behav. 2015; 53:181-8. [DOI:10.1016/j. chb.2015.07.005]

[18] Um YJ, Choi YJ, YooSY. Relationships between smartphone dependency and aggression among middle school students: Mediating and moderating effects of ego-resilience, parenting behavior, and peer attachment. Int J Environ Res Public Health. 2019; 16(19):3534. [DOI:10.3390/ijerph16193534] [PMID] [PMCID]

[19] Yen CF, Tang TC, Yen JY, Lin HC, Huang CF, Liu SC, et al. Symptoms of problematic cellular phone use, functional impairment and its association with depression among adolescents in Southern Taiwan. J Adolesc. 2009; 32(4):863-73.[DOI:10.1016/j. adolescence.2008.10.006] [PMID]

[20] Mehroof M, Griffiths MD. Online gaming addiction: The role of sensation seeking, self-control, neuroticism, aggression, state anxiety, and trait anxiety. Cyberpsychol Behav Soc Netw. 2010; 13(3):313-6. [DOI:10.1089/cyber.2009.0229] [PMID]

[21] Polman H, de Castro BO, van Aken MAG. Experimental study of the differential effects of playing versus watching violent video games on children's aggressive behavior. Aggress Behav. 2008; 34(3):256-64. [DOI:10.1002/ab.20245] [PMID]

[22] Cote KA, McCormick CM, Geniole SN, Renn RP, MacAulay $\mathrm{SD}$. Sleep deprivation lowers reactive aggression and testosterone in men. Biol Psychol. 2013; 92(2):249-56. [DOI:10.1016/j.biopsycho.2012.09.011] [PMID]

[23] Whalen DJ, Gilbert KE, Barch DM, Luby JL, Belden AC. Variation in common preschool sleep problems as an early predictor for depression and anxiety symptom severity across time. J Child Psychol Psychiatry. 2017; 58(2):151-9. [DOI:10.1111/jcpp.12639] [PMID] [PMCID]

[24] Coulombe JA, Reid GJ, Boyle MH, Racine Y. Sleep problems, tiredness, and psychological symptoms among healthy adolescents. J Pediatr Psychol. 2011; 36(1):25-35. [DOI:10.1093/jpepsy/ jsq028] [PMID]

[25] Liu S, Wing YK, Hao Y, Li W, Zhang J, Zhang B. The associations of long- time mobile phone use with sleep disturbances and mental distress in technical college students: A prospective cohort study. Sleep. 2019; 42(2):1-12. [DOI:10.1093/sleep/zsy213] [PMID]

[26] Sahin S, Ozdemir K, Unsal A, Temiz N. Evaluation of mobile phone addiction level and sleep quality in university students. Pak J Med Sci. 2013; 29(4):913-8. [DOI:10.12669/pjms.294.3686] [PMID] [PMCID] 
[27] Exelmans L, Van den Bulck J. Bedtime mobile phone use and sleep in adults. Soc Sci Med. 2016; 148:93-101. [DOI:10.1016/j.socscimed.2015.11.037] [PMID]

[28] Taylor DJ, Bramoweth AD, Grieser EA, Tatum JI, Roane BM. Epidemiology of insomnia in college students: Relationship with mental health, quality of life, and substance use difficulties. Behav Ther. 2013; 44(3):339-48. [DOI:10.1016/j. beth.2012.12.001] [PMID]

[29] Li JB, Lau JTF, Mo PKH, Su XF, Tang J, Qin ZG, Gross DL. Insomnia partially mediated the association between problematic Internet use and depression among secondary school students in China. J Behav Addict. 2017; 6(4):554-63. [DOI:10.1556/2006.6.2017.085] [PMID] [PMCID]

[30] Gaultney JF. The prevalence of sleep disorders in college students: Impact on academic performance. J Am Coll Health. 2010; 59(2):91-7. [DOI:10.1080/07448481.2010.483708] [PMID]

[31] Chen YL, Gau SSF. Sleep problems and internet addiction among children and adolescents: A longitudinal study. J Sleep Res. 2016; 25(4):458-65. [DOI:10.1111/jsr.12388] [PMID]

[32] Lemma S, Gelaye B, Berhane Y, Worku A, Williams MA. Sleep quality and its psychological correlates among university students in Ethiopia: A cross-sectional study. BMC Psychiatry. 2012; 12:237. [DOI:10.1186/1471-244x-12-237] [PMID] [PMCID]

[33] Vail-Smith K, Felts WM, Becker C. Relationship between sleep quality and health risk behaviors in undergraduate college students. Coll Stud J. 2009; 43(3):924-30. https://eric. ed.gov/?id=EJ872309

[34] Männikkö N, Ruotsalainen H, Demetrovics Z, LopezFernandez O, Myllymäki L, Miettunen J, et al. Problematic gaming behavior among Finnish junior high school students: Relation to socio-demographics and gaming behavior characteristics. Behav Med. 2018; 44(4):324-34. [DOI:10.1080/089642 89.20171378608] [PMID]

[35] Nahas M, Hlais S, Saberian C, Antoun J. Problematic smartphone use among Lebanese adults aged 18-65 years using MPPUS-10. Comput Hum Behav. 2018; 87:348-53. [DOI:10.1016/j.chb.2018.06.009]

[36] Barry CT, Loflin DC, Doucette H. Adolescent self-compassion: Associations with narcissism, self-esteem, aggression, and internalizing symptoms in at-risk males. Pers Individ Differ. 2015; 77:118-23. [DOI:10.1016/j.paid.2014.12.036]

[37] Kwon M, Kim DJ, Cho H, Yang S. The smartphone addiction scale: Development and validation of a short version for adolescents. PLoS One. 2013; 8(12):e83558. [DOI:10.1371/ journal.pone.0083558] [PMID] [PMCID]

[38] Lopez-Fernandez O. Short version of the smartphone addiction scale adapted to Spanish and French: Towards a crosscultural research in problematic mobile phone use. Addict Behav. 2017; 64:275-80. [DOI:10.1016/j.addbeh.2015.11.013] [PMID]

[39] Salahuddin M, Maru TT, Kumalo A, Pandi-Perumal SR, Bahammam AS, Manzar MD. Validation of the Pittsburgh sleep quality index in community dwelling Ethiopian adults. Health Qual Life Outcomes. 2017; 15(1):58. [DOI:10.1186/ s12955-017-0637-5] [PMID] [PMCID]

[40] Mollayeva T, Thurairajah P, Burton K, Mollayeva S, Shapiro $\mathrm{CM}$, Colantonio A. The Pittsburgh sleep quality index as a screening tool for sleep dysfunction in clinical and non-clinical samples: A systematic review and meta-analysis. Sleep Med Rev. 2016; 25:52-73. [DOI:10.1016/j.smrv.2015.01.009] [PMID] 